\title{
Fuentes documentales para el estudio de la historia del Norte de Africa existentes en el Archivo de la Presidencia del Gobierno
}

Luisa Auñón Manzanares

Archivo Central. Ministerio Relaciones con las Cortes y de la Secretaría del Gobierno

\section{INTRODUCCION}

El Ministerio de Relaciones con las Cortes y de la Secretaría del Gobierno, creado por Real Decreto 1519/86, de 25 de julio, es el Departamento Ministerial que hoy ha heredado las funciones y las competencias de la Presidencia del Gobierno en lo referido a la Secretaría del Consejo de Ministros. EI R. D. 984/87, de 27 de julio, que determina la estructura básica del Ministerio, hizo depender el Archivo de la Presidencia, de la Secretaría General Técnica del Departamento.

Según la vigente Ley 16/85, de 25 de julio, del Patrimonio Histórico Español, un archivo es (art. 59) un conjunto orgánico de documentos, o la reunión de varios de ellos, reunidos por las personas juridicas, públicas o privadas, en el ejercicio de sus actividades, al servicio de su utilización para la investigación, la cultura, la información y la gestión administrativa.

Dentro del Sistema Archivístico de la Administración (ANEXO 1), el Archivo Central de un Ministerio, como nuestro Centro, es el segundo eslabón del Sistema, y los documentos que en él se conservan están en el segundo estadio o fase de su "vida documental". 
El fondo está constituido por los documentos transferidos desde los diferentes archivos de gestión de las unidades administrativas de ese Ministerio y Organismo, y que con el paso del tiempo se integrarán en el Archivo Intermedio - Archivo General de la Administración Givil del Estado de Alcalá de Henares, creado por D. 914/69, de 8 de mayo-, depósito en el que se deberá decidir, según los plazos establecidos por las disposiciones vigentes, qué documentos pasarán al Archivo Histórico Nacional (último estadio del Sistema) para su conservación permanente, y cuáles deberán ser eliminados, total o parcialmente, una vez creada la Comisión Superior Calificadora de Documentos Administrativos, prevista en el artículo 58 de la Ley del Patrimonio.

Este sería el funcionamiento de un sistema lógico de archivos, basado en las diferentes fases y valores que el documento va adquiriendo con el paso del tiempo; pero la realidad es que rara vez se cumplen estos plazos y nuestro Centro, aunque periódicamente transfiere documentación al Archivo General de Alcalá, tampoco es una excepción.

En el Archivo de Presidencia custodiamos, por decisión de las Autoridades responsables del Departamento, documentación que debería estar depositada en el Archivo General de la Administración de Alcalá o en el Archivo Histórico Nacional, y que, lógicamente, es la de mayor valor histórico.

Para entender la organización del archivo de una Institución o Departamento, y la razón por la que en él esté depositada determinada documentación, es imprescindible conocer la evolución histórico-legislativa de esa Institución.

\section{LA PREBIDENCIA DEL CON8EJO DE MINISTROS: GENERIS Y EvoLUcrow}

La Presidencia es desde sus comienzos un Centro difuso de competencias, que abarca objetivos que van desde la dirección estrictamente política del país, a la coordinación e impulso de todos los sectores de la acción administrativa.

La presidencia del Gobierno representa al Gobierno de la Nación, dirige su política general y asegura la coordinación de todos los órganos de Gobierno y administración.

Al observador objetivo de la Presidencia, se le plantea el problema de pensar, qué razones fueron motivando a lo largo de la Historia, el 
que diferentes organismos se hayan integrado en ella. Para resolverlo habría que estudiar exhaustivamente la Legislación, que en determinadas ocasiones se ocupó más de detallar instituciones marginales, que de institucionalizar y estructurar esquemas orgánicos de carácter definitivo.

Quizás nuestro recorrido para estudiar las diferentes disposiciones que afectaron a los organismos productores de la documentación que nos ocupa, no pueda evitar, aunque lo intentemos, contagiarse del desorden y la falta de continuidad de la Institución.

La unidad de concierto en la adopción y ejecución de las medidas de Gobierno, constituye una necesidad imperiosa cuando la responsabilidad de gobernar se distribuye entre varias personas. De ahí que el Real Decreto de 19 de noviembre de 1823 (dirigido por Fernando VII a Víctor Sáez, Secretario de Estado y del Despacho de Estado), por el que los Secretarios de Estado y del Despacho formaron un Consejo de Ministros, para tratar todos los asuntos de interés y utilidad general, configuró una Institución jurídica equivalente al Gobierno.

Para entender esta disposición, hay que recordar que aunque Fernando VII había abolido, en octubre de 1823, la Constitución de Cádiz de 1812, lo cierto es que su espíritu estaba en toda la legislación de la época.

Aunque el párrafo XXIX del Discurso preliminar de la Constitución de 1812 , habla de la responsabilidad del Gobierno en ninguno de sus artículos existe la idea de Gobierno en el sentido de institución colegiada, ni por supuesto tampoco, de la Presidencia como organismo independiente.

Lo mismo se puede deducir de las intenciones del Monarca: tras el preámbulo del Decreto de 19 de noviembre, el Rey se limita a configurar el Consejo como organismo en el que cada Ministro dará cuenta de los negocios correspondientes a la Secretaría de su cargo, recibirá mis resoluciones y cuidará de hacerlas ejecutar.

El Consejo de Ministros es en este momento sólo un instrumento de consulta y asesoramiento, pero será también, una Institución básica para nuestro sistema político-administrativo, que sólo dejará de existir en algunos momentos en que el propio sistema se quiebra, por acontecimientos de su propia evolución, y eso, sólo, de manera transitoria, aún dentro de las nuevas formas de gobierno del Estado. 
Esta situación anómala tuvo lugar en dos períodos históricos: 15 de septiembre de 1923 a 3 de diciembre de 1925 (Directorio Militar) y 18 de julio de 1936 a 30 de enero de 1938 (aunque en este momento no se habla de Consejo de Ministros, sino de Cobierno de la Nación).

Durante la primera etapa del Consejo de Ministros, no existe formalmente un Presidente de Gobierno, hasta el R. D. de 31 de diciembre de 1824, en el que el Rey dispone que su Primer Secretario de Estado y de Despacho Universal... y los que lo fuesen en lo sucesivo presidirán el Consejo en ausencia del Rey. Pero no es hasta 1832, que se puede considerar a Cea Bermúdez como Primer Presidente del Consejo.

El R. D. de 18 de abril de 1834, promulga un texto que la Historia conoce como el Estatuto Real. Es una fórmula de compromiso entre los Tradicionalistas y los Liberales de Isabel II que querían la vuelta de la Constitución de Cádiz. Va dirigido a Martínez de la Rosa, Presidente del Consejo de Ministros, nuevamente el legislador fija un Organismo, la Presidencia, sin una estructura previa consolidada.

La configuración de Presidencia será fruto de una Administración Pública moderna y eficiente.

El R. D. de 15 de abril de 1871, es fundamental en nuestro recorrido por que da estado oficial a la documentación de la actividad del Consejo de Ministros, de la que hasta ese momento, no existía constancia alguna. En la exposición de motivos se detalla: La importancia y variedad de los acuerdos que de ordinario se toman en el Consejo de Ministros demuestran la conveniencia de que se levanten actas formales.

Se crea el cargo de Secretario del Consejo de Ministros, que recae en el Subsecretario Ordenador General de Pagos de la Presidencia.

Es curioso pensar que en el momento en que el legislador crea el cargo de Secretario del Consejo de Ministros, dejamos de tener testimonio escrito, es decir: Actas y Acuerdos, de los asuntos tratados en el mismo, hasta diciembre de 1925.

EI R. D. de 23 de abril de 1890, que promulga el Reglamento Provisional de Procedimiento Administrativo de la Presidencia del Consejo de Ministros, es, podríamos decir, "la Partida de Nacimiento" del complejo Organismo que estudiamos.

La Presidencia deja de ser una Institución "de facto" para entrar a disfrutar "de iure" de una imprescindible estructuración. 
A través de este Reglamento de Procedimiento Administrativo se hace oficial y legítimo el trámite por Presidencia de toda clase de problemas administrativos y de los asuntos que no constituyen expediente, que no son más que una especie de "correspondencia oficial" con los demás poderes del Estado.

Con el paso del tiempo esta "correspondencia oficial" va a tener una importancia fundamental, y en ella quizás, se pudiera encontrar el origen de esa labor coordinadora, de que hemos hablado, y que hoy se le atribuye a la Presidencia del Gobierno.

En una pirueta temporal - y sin detenernos a analizar las incidencias de la Legislación sobre Funcionarios y temas económicos, de 1907 a 1918, que afectaron profundamente a la Presidencia, pero que exceden del interés de este trabajo-, vamos a aterrizar en un momento político y administrativo fundamental en nuestra Historia: el Directorio Militar.

De septiembre de 1923 a enero de 1930, y por la naturaleza del régimen político implantado, sufren los servicios de la Presidencia del Consejo de Ministros diversos avatares, no siempre reflejados en los textos legales.

Por los Reales Decretos de 15 de septiembre de 1923, el Teniente General Primo de rivera, recibe los nombramientos de Jefe de Gobierno y Presidente del Directorio Militar. Se suprimen los cargos de Presidente del Gobierno y Ministros de la Corona, y los de Subsecretarios de la Presidencia y de los demás Departamentos, salvo Guerra y Estado. Se suprime el cargo de Presidente del Consejo, por el afán de eliminar toda clase de cargos políticos, pero no la dependencia: Presidencia.

En diciembre de 1925 se modifica el sistema de Gobierno, por Real Decreto de 3 de diciembre, se sustituye el Directorio Militar por un Gobierno de carácter Civil, que restablece los cargos de Presidente y Ministros de la Corona, y nombra a Miguel Primo de Rivera, Presidente del Consejo de Ministros.

El Real Decreto-Ley de 15 de diciembre de 1925 determina el esquema orgánico de la Presidencia del Consejo de Ministros, en el que entre otros organismos adscritos, dependientes del Jefe del Gobierno está la Dirección General de Marruecos y Colonias, que absorbió las funciones de la Oficina de Marruecos, creada por Presidencia en 1924, y las de la extinguida Sección Colonial del Ministerio de Estado (ANEXO 2). En esta ads- 
cripción a la Presidencia de los Asuntos de Marruecos está presente el centralismo del Presidente del Gobierno que quiere tener de la mano, toda la política colonial; postura ésta ratificada por el Real Decreto-Ley de 3 de noviembre de 1928, que suprimió el Ministerio de Estado y lo integró en el de Presidencia, para poder llevar directamente las relaciones exteriores el jefe del Gobierno.

El 30 de enero de 1930, el Rey acepta la dimisión de Primo de Rivera para dar paso a un Gobierno presidido por Berenguer y más tarde por Aznar, cuya norma de conducta fue la de deshacer la labor del Directorio.

Estos acontecimientos marcan el final de una etapa histórica y el principio de los días de la Segunda República en cuya labor administrativa nos vamos a detener.

La Constitución de 9 de diciembre de 1931, es el primer texto a considerar: El Título V está dedicado al Presidente de la República, que "es el Jefe del Estado y personifica a la Nación" y que en el artículo 75 tiene como competencia el nombramiento del Presidente del Consejo de Ministros, y a propuesta de éste los Ministros.

Los Decretos de 19 de julio, 26 de julio y 11 de diciembre de 1934, modifican la denominación de la Dirección General de Marruecos y Colonias, creando primero la Secretaría Técnica de Marruecos y Colonias, y después dos organismos centrales asesores que son. la Inspección General de Colonias y la Junta Asesora Jurídica de Colonias. Es simplemente un mero juego terminológico, de un régimen cuyo final se acercaba velozmente.

Los Gobiernos de Lerroux, Chapaprieta y Portela, cada uno de ellos en dos ocasiones, intentan dirigir la República Española. Las crisis se suceden y también las disposiciones contradictorias, que repercuten directamente en los Organismos de nuestro interés; La Inspección General de Colonias da paso a una Secretaría General de Colonias, y ésta, en cuestión de meses, por Decreto de 31 de diciembre de 1935, se refunden la Secretaría Técnica de Marruecos, en un organismo denominado Dirección General de Marruecos y Colonias.

Los acontecimientos de 18 de julio de 1936, produjeron hondas transformaciones en la estructura de la Administración Central del país.

El Decreto de 24 de julio de 1936, crea la Junta de Defensa Nacional que "asume todos los poderes del Estado y representa legítimamente al país ante las potencias extranjeras". Tiene una vida efímera ante 
la necesidad de concentrar en una sola mano tareas de guerra y gobierno. El 29 de septiembre de 1936, la Junta de Defensa Nacional nombra Jefe de Gobierno del Estado Español al General Franco, quien asumió todos los poderes del Nuevo Estado.

La Ley de 1 de octubre de 1936, va a suponer una regulación administrativa, a través de la creación de la Junta Técnica del Estado, que se puede entender como "un sucedáneo" del Gobierno que no era posible crear aún. Esta Junta se ocupó de las funciones administrativas del Nuevo Estado; se dividió en siete secciones o Comisiones: Hacienda, Justicia, Industria, Comercio y Abastos, Agricultura y Trabajo Agrícola, Trabajo, Cultura y Enseñanza, y Obras Públicas y Comunicaciones, y fue reglamentada orgánicamente el 19 de noviembre de 1936.

El esquema de la Junta corresponde, sin lugar a dudas, al de un Gobierno "de urgencia" que cumple algunas tareas mientras el país está en una situación bélica. Es un órgano consultivo que somete sus dictámenes al Jefe del Estado.

En 1938 las exigencias de una realidad política y social cada vez compleja, aconsejaron promulgar la Ley de 30 de enero de 1938, por la que la Administración Central del Estado se organiza en Departamentos Ministeriales subordinados a la Presidencia del Gobierno (una aportación interesante del Derecho Positivo, ya que de esta Ley parte una organización que, aunque se haya ampliado y modificado en cuanto al número y competencias de los Ministerios, no ha cambiado en lo esencial).

Se crea el cargo de Vicepresidente, del que dependerán directamente: La Subsecretaría de la Presidencia, el Instituto Geográfico y Estadístico, el Servicio de Abastecimientos y Transportes y el Servicio de Marruecos y Colonias.

Esta Ley da carta de naturaleza al sentido gestor de la Presidencia y crea la Vicepresidencia para aligerar la carga burocrática que recaería en otro caso, en el Presidente del Gobierno, que es a la vez Jefe de Estado.

La Legislación de guerra finaliza con la Ley de 29 de diciembre de 1938, que sirve para afianzar la superioridad jerárquica de la Presidencia.

"Terminada la guerra y comenzadas las tareas de reconstrucción y resurgimiento de España... aconseja una acción más directa y personal del Jefe del Estado", estos dos párrafos entresacados del preámbulo de la Ley de 8 de agosto de 1939, suponen un nuevo planteamiento de la vida 
española e inciden directamente sobre nuestra Institución: Se suprime la Vicepresidencia del Gobierno, pasando a depender del Presidente los organismos y funciones que dependían de ella, a excepción de la Dirección General de Marruecos y Colonias que pasará a formar parte del Ministerio de Asuntos Exteriores.

Se configura la Presidencia como un Ministerio y se le asigna un Subsecretario. Se da un concepto más operativo a la Presidencia, que sólo se va a ocupar de la política general y va a ser Centro coordinador, sino que tendrá a su cargo otras funciones.

La estructura básica de la Administración Central se mantenía inalterada desde la Ley de 8 de agosto de 1939, pero la compleja actividad administrativa, exigía una reorganización de los servicios centrales.

El Decreto-Ley de 19 de julio de 1951 entre otras innovaciones tuvo una trascendental: la elevación del rango del titular de la Subsecretaría de Presidencia, convirtiéndolo en Ministro Subsecretario de la Presidencia (artículo $4^{\circ}$ ), el cual será además Secretario del Consejo de Ministros, sustituyendo a Ramón Serrano Suñer, Ministro de Gobernación (2-2-1938 a 2-10-1940) y a José Ibáñez Martín, Ministro de Educación (2-10-1940 a 19-7-1951) que habían desempeñado el cargo anteriormente.

Aunque el cargo de Presidente se reguló con total independencia del de Jefe del estado, en la Ley de Régimen Jurídico de la Administración del Estado, de 20 de julio de 1957, no es hasta la Ley Orgánica del Estado, de 10 de enero de 1963, cuando se produce la definitiva separación entre la Jefatura del Estado y la Presidencia del Gobierno, aunque, provisionalmente los dos cargos siguieran recayendo en el mismo titular, Francisco Franco.

El 21 de septiembre de 1967, fue nombrado Vicepresidente del Gobierno Luis Carrero Blanco, a quién como Ministro Subsecretario de la Presidencia, hemos visto correspondía el cargo de Secretario del Consejo de Ministros y de las Comisiones Delegadas del Gobierno. Por una cierta incompatibilidad entre estas dos funciones y las de Vicepresidente del Gobierno, por Decreto de la Jefatura del Estado de 5 de octubre, delegó las funciones de Secretario del Consejo de Ministros en el Ministro de Información y Turismo.

Por Decreto 1.145/73, de 8 de junio, Carrero Blanco fue nombrado Presidente del Gobierno. 
La Ley de 2 de enero de 1974 produjo el cambio de nombre de Ministro Subsecretario de la Presidencia, por el de Ministro de la Presidencia, aunque denominando al Departamento Presidencia del Gobierno. Situación ésta que se mantuvo hasta 1977, en que, por Real Decreto de 4 de junio, recibe el nombre de Ministro de la Presidencia, y deja de ser un Departamento especial vinculado al Jefe del Estado.

El Decreto de 28 de octubre de 1977 integra el Ministerio en el conjunto institucional de la Presidencia, sufriendo el Departamento una pérdida de su propia sustantividad.

El Real Decreto-Ley de 7 de diciembre de 1982 y la Ley de 16 de agosto de 1983, contemplan una Presidencia del Gobierno integrada por un Presidente del Gobierno y unos órganos encargados de asistirle en el ejercicio de sus funciones, y entre ellos el Ministerio de la Presidencia.

En el Real Decreto 1.519/86, de 25 de julio, por le que se reestructuran los Departamentos Ministeriales, desaparece "cautelosamente" el Ministerio de la Presidencia, sin que se de ninguna explicación sobre ello (el legislador ha sido capaz de borrar de un plumazo la señal de una Institución, sin explicar el por qué).

Se crean en este Real Decreto el Ministerio de Relaciones con las Cortes y de la Secretaría del Gobierno, y el Ministerio para las Administraciones Públicas, que asume algunas competencias que correspondían al Ministerio de la Presidencia (entre otras las de Comisión Liquidadora de Organismos: Oficina Gestora de la documentación de la extinguida Comisión para la Transferencia de los Intereses Españoles en el Sáhara).

FI ARCHIVO DE LA PAESIDFITCIA DEL GOBIBHNO: ORIGENES Y FONDOB PARA FL EBTUDIO DE LA HISTORIA DEL NORTE DE AFRICA

Las referencias al Archivo del Consejo de Ministros como órgano diferenciado, no aparecen hasta muy avanzada la segunda mitad del XIX: el 26 de enero de 1871, a propuesta del Presidente Serrano, que comisiona a uno de los funcionarios para recoger la documentación del Consejo que se encontraba depositada en el Archivo del Ministerio de Estado (de esta fecha es el primer Inventario de documentación que se conserva en el Archivo).

Los fondos custodiados no se remontan más allá de 1824, año en el que se inicia la documentación más valiosa del Centro: Las Actas y 
Acuerdos de los Consejos de Ministros, verdadero arsenal de datos para la Historia Contemporánea, especialmente hasta 1844.

El fondo del Archivo está organizado por procedencias: Secciones y Series documentales, es decir, por conjuntos de documentos con un mismo origen orgánico, con idéntica función administrativa y con unas características externas y de contenido semejantes. Hay Secciones cerradas y abiertas -modificadas por los cambios estructurales y competenciales-, que se van enriqueciendo con la documentación remitida por las diferentes Unidades del Departamento.

Hay, además, cuatro fondos del Archivo General de la Administración de Alcalá de Henares, que custodian documentación dependiente de nuestro Departamento:

Presidencia, Sindicatos, Cultura (de éste sólo la documentación de Sección Femenina) y Africa.

El Centro, además de los trabajos que habitualmente se desarrollan en los Archivos, tiene dos aspectos determinantes de su función: es un Archivo Administrativo que recoge la documentación producida por las Unidades del Departamento a las que sirve de apoyo en el desarrollo de su gestión, y custodia un fondo muy importante para la Historia de España de los XIX y XX. Por todo ello, el Archivo de la Presidencia constituye un importante centro de investigación histórica, tanto por su fondo estrictamente documental, como por el histórico-bibliográfico que le sirve de apoyo.

Vamos a pormenorizar las series documentales de más interés para el estudio de la Historia de España Contemporánea y que inciden directamente en el tema de este Seminario.

Estos fondos no están constituidos por expedientes que formalmente se puedan considerar como fruto de una gestión administrativa, pero sí, por una documentación muy importante desde el punto de vista histórico.

\section{Actas del Consejo de Ministros}

Son la primera piedra de lo que hoy es el Archivo de la Presidencia del Gobierno. No es un fondo excesivo en cuanto a su volumen pero sí muy importante por su contenido.

Lo constituyen 18 Libros de Actas originales y Acuerdos del Consejo de Ministros de los años 1824 a 1930, con muy importantes lagu- 
nas históricas: Primera República, Restauración, primera década del siglo $\mathrm{XX}$; y 22 legajos con documentación complementaria de los mismos años, aunque en estos legajos hay noticias históricas, no recogidas en Libros (se conservan notas de las reuniones del Consejo de 1874, con el membrete de Presidencia del Poder Ejecutivo, y de las que sin embargo no existe Libro de Actas formalizado). (ANEXO 3).

\section{Actas del Consejo de Ministros, 1835 a 1856}

Esta documentación, consultada por numerosos investigadores, corresponde a las deliberaciones, en materia nacional e internacional mantenidas por los Secretarios de Estado y del Despacho y los Monarcas.

A través de estas minuciosas Actas, conocemos las deliberaciones, dictámenes y acuerdos del Consejo; son imprescindibles para contrastar datos y averiguar y estudiar hechos de la Historia de España y sus relaciones internacionales durante el siglo XIX.

Vamos a destacar algunos asuntos concernientes a la acción de España en el Norte de Africa, de los que las Actas nos dan la noticia:

- Consecuencias del Tratado de Túnez en 1791, sobre introducción de productos españoles en aquella Regencia.

- Arbitraje francés en los asuntos de España con Argel.

- Relaciones de España con el Rey de Argel.

- Tratados con Marruecos sobre revolucionarios españoles en ese país.

- Relaciones con Marruecos.

- Situación de españoles en Túnez, Francia y Argel.

- Créditos del Gobierno.

- Tráfico de negros.

- Actuación de buques corsarios en el Mediterráneo.

- Redención de cautivos.

- Prisioneros insurgentes en Ceuta.

- Desertores de Ceuta.

- Presidios del Norte de Africa.

- Pesca en Ceuta y Melilla.

- Obligaciones con Argel.

- Medidas de Orden Público en la zona mora de Ceuta.

- Disminución del número de presidios de Alhucemas. 
- Comandantes de los Presidios.

- Alianzas de España con Inglaterra, Francia y Portugal.

Como es evidente, por el tipo documental que forma esta serie, todos estos asuntos no se tratan exhaustivamente, pero es en estas Actas donde se indica el Departamento Ministerial en el que se pueden encontrar los documentos, por ser de su competencia el asunto de que se trata. Documentos que, por su fecha, pueden estar depositados ya en el Archivo Histórico Nacional.

El Ministerio, a través de nuestro Archivo, está llevando a cabo la Edición de Fuentes de este material hasta la Dictadura de Primo de Rivera.

Los cuatro primeros volúmenes de los años 1824 a 1829, están ya a disposición del gran público. La edición consta de una Introducción histórica, Organigramas de los diferentes Gobiernos, Glosario de abreviaturas, Transcripción del texto original de las Actas, Selección de documentos originales representativos e Índice Onomástico, Toponímico y de Conceptos.

Actas del Gabinete civil de la dictadura de Primo de Rivera, 1925 a 1930

De este período histórico tan importante para el estudio de Africa, en el Archivo Central sólo custodiamos las Actas a las que ya hemos hecho mención y algunas disposiciones emanadas de la Presidencia del Directorio, ya que el grueso del fondo documental, fue transferido, en el año 1969, al Archivo Histórico Nacional.

Cuando la gestión de Primo de Rivera llega a su fin, en su activo se pueden apuntar: El final de la guerra de Marruecos, la restauración del Orden Público, el final del terrorismo, la red de carreteras y ferrocarriles y un vasto programa de obras públicas de todas las especies.

No es mi intención, ni soy la persona adecuada, llegar hasta el fondo de estas realidades; es mi objetivo simplemente, presentar, a través de las Actas, las intenciones del Directorio, cualquiera que hoy sea nuestro juicio sobre él.

En un análisis exhaustivo de las 288 Actas de este período encontramos información sobre:

- Condiciones de Abd-el-Krim al Gobierno francés para su sumisión. 
- Armisticio solicitado a francia por Abd-el-Krim.

- Rendición de Abd-el-Krim.

- Confinamiento de Abd-el-Krim.

- Sometimiento de las Kabilas de Tetuán y Yebala.

- Rendición de la Kabila de Anyera por el Ejército.

- Indultos a moros.

- Partidas de la Kabila de Ketama.

- Actitudes favorables de Francia sobre Marruecos.

- Conversaciones en Madrid con el Mariscal Petain sobre las operaciones de Marruecos.

- Situación del Ejército de Marruecos.

- Proyecto de Convenio Hispano-Francés del General Jordana sobre Marruecos.

- Estatuto de Tánger e intervención de Italia favorable a España.

- Ordenanzas de Aduanas en los puertos francos de Ceuta y Melilla.

- Comercio en la Zona del Norte de Africa.

- Adquisición de barcazas para el transporte y vertido, de las escolleras del puerto de Melilla.

- Reacciones contra España de los marroquíes en el sector de Melilla.

- Visita de los Ministros del Gabinete a la Cala de Quemado, Melilla y Ceuta para evaluar desastres por los temporales sufridos en la zona.

- Suscripción pública para paliar los efectos de los temporales en la Zona Española de Africa.

- Reorganización de los mandos miliıares en Melilla, Ceuta-Tetuán, Axdir y Larache.

- Adscripción de las Islas Chafarinas a la circunscripción de Melilla.

- Mando civil en las Plazas de Soberanía y constitución de Comisiones Mixtas y Administrativas en Ceuta y Melilla.

- Viaje del Presidente del Gobierno a Marruecos.

- Ferrocarril Tánger-Fez.

- Viaje de SS.MM. y su séquito a Marruecos. 
- Acuerdos sobre apertura de navegación aérea en la zona prohibida de Ceuta.

- Plan General de Obras Públicas en Marruecos: Carreteras, puentes, vías férreas, organismos agrícolas e industriales, etc.

- Proyectos de obras en la Zona de Villa Sanjurjo.

- Estatuto General del Personal al servicio de la Administración de la Zona del Protectorado de España en Marruecos.

Como hemos advertido las Actas sólo nos dan la noticia de lo tratado por el Gobierno en su conjunto. Estos documentos del Gabinete Civil son menos prolijos en detalles que los de la época fernandina, sólo recogen lo dispuesto, el acuerdo, pero no las deliberaciones mantenidas por el Gobierno en el seno del Consejo de Ministros.

Todo este conjunto documental, ya preparado por el Archivo, espera ver la "luz editorial" el próximo mes de julio siguiendo el plan del Ministerio.

\section{Presidencia del Gobierno}

Junta de Defensa Nacional, Junta Técnica del Estado, Presidencia y Vicepresidencia

Ya hemos apuntado que el Decreto de 24 de julio de 1936, crea una Junta de Defensa Nacional, de carácter militar, presidida por el General Cabanellas, que asumió todos los poderes del Estado. Sustituida en la Ley de $1^{\circ}$ de octubre de 1936 por la Junta Técnica del Estado, que subsistió hasta enero de 1938.

El 29 de septiembre de 1936 la Junta dicta un Decreto por el que se nombra Jefe del Gobierno del Estado al General Franco, haciéndole entrega de los plenos poderes de su magistratura el $1^{\circ}$ de octubre, en la Capitanía General de Burgos.

La documentación de estas instituciones se custodia en la misma Serie documental, y es fundamental para el estudio de la creación, evolución y asentamiento del Nuevo Estado franquista.

Es una documentación estructurada por Organismos; abarca 38 legajos de documentación de los años 1936 a 1947 con asuntos sobre: Personal que constituyó las Juntas, Disposiciones emanadas de la Junta, 
Asuntos Coloniales, Informes de las distintas Comisiones, Correspondencia del Jefe del Estado, etc.

En el legajo núm. 18, y bajo el epígrafe de MARRUECOS Y COLONIAS, podemos encontrar documentación de los años 1938 y 1939 referida a:

- Personal de Marruecos y Colonias.

- Ley de conversión de las obligaciones españoles del ferrocarril Tánger-Fez.

- Correspondencia con el Gobernador de Guinea.

- Correspondencia sobre el Consulado Español de Tánger.

- Gréditos a la Alta Comisaría de España en Marruecos.

\section{Jefatura del Estado}

Esta serie la constituyen 60 legajos de documentación, recogidos en el Palacio de El Pardo, a la muerte del anterior Jefe de Estado.

Son documentos recibidos por Franco, no en su calidad de Jefe del Estado, sino de Presidente del Gobierno, dualidad que se mantuvo hasta 1973 (nombramiento de Luis Carrero Blanco) de "iure", aunque de "facto" desde 1967, Carrero Blanco, como Vicepresidente del Gobierno ejercía funciones de Presidente.

No son expedientes administrativos propiamente dichos sino telegramas cifrados y numerados, informes y documentos para asesoramiento y conocimiento de la Jefatura del Estado.

La documentación está estructurada por Departamentos Ministeriales, siendo imprescindibles como Fuente, para el tema que nos ocupa, los siguientes Ministerios:

- Ministerio de Asuntos Exteriores: años 1938-1959 (legajos 1 al 34).

La documentación custodiada procede de las diferentes Embajadas, Consulados y Legaciones de España en Europa, América, Africa y Extremo Oriente.

Contiene: Telegramas cifrados y numerados; informes y comunicaciones de la Oficina de Información Diplomática; Cartas y otros tipos documentales sobre asuntos confidenciales de política internacional, ordenados cronológicamente. 
- Ministerio del Ejército. IV. Alto Estado Mayor: años 1940-1953 (legajo 39).

Con información sobre el Ejército Colonial francés en Marruecos.

- Presidencia: Marruecos y Colonias: años 1938-1948 (legajo $60)$.

Contiene, entre otras, documentación sobre los siguientes temas:

- Manifiesto de Tánger.

- Movimientos nacionalistas.

- Mapas del Protectorado.

- Alta Comisaría de España en Marruecos: Cartas de Franco, Gartas del General Varela, documentos sobre el viaje del Sultán a Tánger, créditos presupuestarios, etc.

\section{Secretaria del Ministro Subsecretario}

Serie integrada por la documentación procedente de la Secretaría de D. Luis Carrero Blanco, que abarca desde su elevación del rango de titular de la Subsecretaría, al de Ministro Subsecretario, por D. L. de 19 de julio de 1951, hasta su nombramiento como Presidente del Gobierno el 8 de julio de 1973.

Comprende 64 legajos de documentación, en gran parte 'lisificada de origen; por el momento no es accesible a la investigación. de acuerdo con los supuestos establecidos en la Ley 16/85, del Patrimonio Histórico Español, y se encuentra en período de estudio.

En este fondo hay 6 legajos de documentación de los años 1942 a 1959 sobre:

- Dirección Ceneral de Marruecos y Colonias.

- Africa Occidental Española.

- Boletines de Información.

- Memorias del Ejército.

- Apresamiento de buques.

- Comercio inglés en la Zona de Marruecos.

- Compañía de Ferrocarriles Tánger-Fez.

- Decretos.

- Operaciones.

- Informaciones de prensa extranjera. 
Estas series documentales están inventariadas e indizadas, lo que facilita enormemente el acceso de los investigadores a la documentación.

\section{Otras series documentales}

\section{Planes Provinciales}

En 1961, el 14 de enero, se crea el Servicio Central de Planes Provinciales, con la misión de tramitar asuntos relacionados con el funcionamiento de planes provinciales de obras y servicios, vigilando su ejecución e informando a la Comisión Delegada del Gobierno para Asuntos Económicos.

Como resultado de esta función, el Archivo custodia en su fondo del Archivo General de la Administración, 179 legajos de Planes Provinciales en los que se encuentran incorporados Planes de las Provincias Africanas de 1959 a 1967. Forman estos expedientes la Memoria del Plan y los diferentes informes del Servicio hasta su finalización.

\section{Personal}

Dentro de la Sección de Personal de la Presidencia, en la Serie de Cuerpos a Extinguir, están depositados los expedientes administrativos de la Escala Auxiliar del Cuerpo de Interpretación árabe y beréber, de la Zona Norte de Marruecos, de los años 1959-1960.

En la Serie de Plazas no escalafonadas hay expedientes administrativos de Delineantes y Topógrafos de la Zona Norte de Marruecos (1).

\section{CONGLUSTON}

Como conclusión de todo lo expuesto, incidir simplemente en dos aspectos:

- La importancia de nuestro Archivo como custodio de una documentación, no excesiva en su volumen, pero sí fundamental para cualquier estudio o investigación, por proceder de una Institución tan peculiar y relevante como la Presidencia del Gobierno, centro de los entresijos de la acción del Gobierno y del funcionamiento del aparato del Estado.

- Las Actas y Acuerdos del Consejo de Ministros, como llave fundamental para el estudio de la Historia de España y de sus relaciones internacionales. 
En el caso de Africa, a la importancia de los fondos documentales custodiados, se suman los indicios que estos documentos aportan sobre otras fuentes claves para el investigador.

Finalmente quiero señalar un aspecto importante, sino fundamental de nuestra función de archiveros: el Servicio a os ciudadanos e investigadores.

Cuando en 1984 el profesor Víctor Morales Lezcano publicó su libro "España y el Norte de África: El Protectorado en Marruecos (1912-1956)", apuntaba en su introducción, entre otros factores objetivos que le habían impedido ofrecer al lector algo más que un cuadro orientativo, en primer lugar el factor archivístico.

El gran párrafo dedicado al tema archivos, estaba quizás sobredimensionado, pero no era más que un claro reflejo de su "lucha" documental.

El tiempo ha pasado, los medios humanos y materiales no se han incrementado todo lo que deberían, y los primeros en sentirlo somos los archiveros, pero ya tenemos un marco de actuación: La Ley 16/85 de 25 de julio, del Patrimonio Histórico Español, y la esperanza de que la creación de la Comisión Superior Calificadora de documentos Administrativos y el aumento de medios humanos, propicie, todavía más, la intensa colaboración que existe y debe existir entre Investigadores y Archiveros. 


\section{Axพ8X 1}

\section{EgTRUCTURA ORGAJICA DEL 8ISTHEA ARCHTVIBTICO}

DE LA ADECHISTRAOTOW

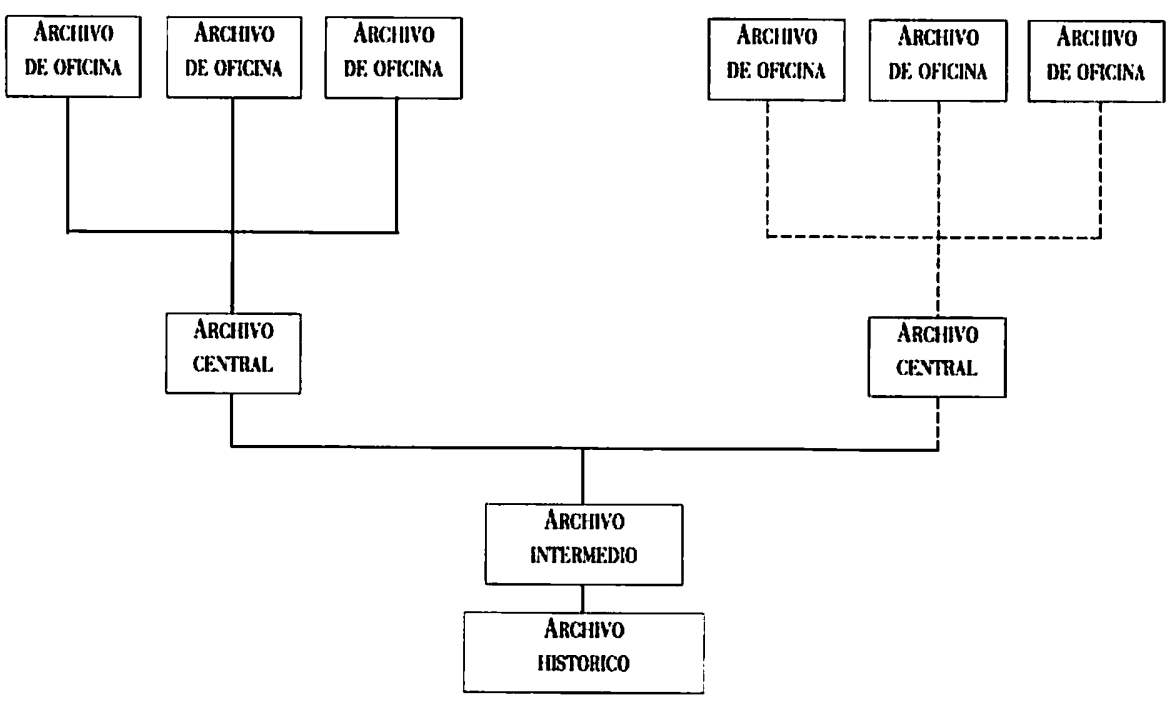

\section{ANBXO \&}

Los Organismos que en la Administración Central han tenido a su cargo la acción de España en el norte de Africa, han sufrido numerosas vicisitudes a lo largo del tiempo. De algunos de ellos ya hemos hecho referencia en el curso de nuestra exposición, y ahora sólo nos resta a modo de anejo, hacer alusión a otros, no señalados, que también han intervenido en estas materias.

La supresión del Ministerio de Ultramar, después de la derrota de 1898 y la convergencia del Consejo de Ministros y la Cartera de Estado en la persona de Silvela, motivaron que la Administración de las Islas de Fernando Poó, Carolinas y Palaus, pasen a Presidencia en 1889.

En 1915 en Decreto de 4 de enero, se publica el Reglamento Interno de la Presidencia, y se crea por otro Real Decreto de 17 de junio la Intervención Civil de Guerra y Marina del Protectorado de Marruecos. 
En 1925 el Real Decreto -Ley de 15 de diciembre, determina el esquema orgánico de la Presidencia y vuelve a ella bajo una Dirección General de Marruecos y Colonias, que absorbió las funciones de la Oficina de Marruecos creada en Presidencia en 1924 y las de la extinguida Sección Colonial del Ministerio de Estado.

En 1938 por Decreto de 8 de febrero, la Vicepresidencia del Gobierno se compone de una Subsecretaría, el Instituto Geográfico y Estadístico, Abastecimientos y Transportes y el Servicio de Marruecos y Colonias. Se divide este Servicio en dos Secciones: una en Marruecos y otra de Colonias, que pasó a depender del Ministerio de Asuntos Exteriores por Ley de 8 de agosto de 1939.

En 1942 la Ley de 15 de enero, adscribe a la Presidencia del Gobierno la Dirección General de Marruecos y Colonias.

La declaración Hispano-marroquí de independencia de la Zona española del Protectorado, hizo que por Decreto de 21 de agosto de 1956, se agruparan todos los servicios de esa Dirección General, bajo la denominación de Dirección General de Plazas y Provincias Africanas, órgano a través del cual la Presidencia del Gobierno ejerció cuantas facultades le concernieron en orden a la Legislación general de los servicios públicos de los Territorios.

También en 1956, y en cumplimiento del Decreto anterior por orden de 26 de septiembre, se produjo la estructuración de la Dirección General de Marruecos y Colonias en diferentes Servicios y Secciones.

En 1965 el Decreto de 15 de febrero, reestructuró la Dirección General de Plazas y Provincias Africanas en dos unidades: Secretaría General y Jefatura Superior de Servicios Financieros.

En 1969 por Decreto de 5 de diciembre, adoptó la denominación de Dirección General de Promoción del Sáhara; extinguida por Real Decreto 680/66 de 8 de abril, que crea la Comisión para la transferencia de los Intereses Españoles en el Sáhara (a la que quedan adscritos los Servicios de la Dirección General de Promoción del Sáhara).

\section{Comisión Liquidadora de Organismos:}

Se crea por Decreto $1.555 / 59$, de 12 de septiembre. 
Se reorganiza por Real Decreto 2.183/80, de 15 de octubre, añadiéndosele las funciones y cometidos de la Comisión para los Intereses Españoles en el Sáhara.

Este Organismo se integró en el Ministerio para las Administraciones Públicas por Real Decreto 1981/86, de 25 de septiembre.

\section{ANBXO 3 \\ CONSERVADAB EN DL ARCHIVO CENTRAL DS LA PRESI- DEITCIA, MIMISTERIO DE RELACIONES CON LAS CORTES Y DE LA SECRETARIA DEL GOBIFRNO}

Libros

Sesiones

de Actas

I 15 de febrero de 1824 (1) a 12 de marzo de 1825.

II 26 de marzo de 1825 a 20 de septiembre de 1825 .

III 23 de septiembre de 1825 a 28 de diciembre de 1825.

IV 19 de septiembre de 1826 a 29 de diciembre de 1827 .

V $\quad 2$ de enero de 1828 a 27 de diciembre de 1828.

VI 3 de enero de 1829 a 30 de diciembre de 1829 .

VII 2 de enero de 1830 a 29 de diciembre de 1830 .

[VII-2] 1 de enero de 1831 a 31 de diciembre de 1831 .

VIII 7 de enero de 1832 a 30 de diciembre de 1832 .

IX 1 de enero de 1833 a 29 de diciembre de 1833.

X 1 de enero de 1834 a 20 de octubre de 1838.

XI 9 de diciembre de 1843 a 24 de abril de 1844 .

XII $\quad 30$ de noviembre de 1854 a 12 de noviembre de 1855 .

XIII 3 de diciembre de 1925 a 24 de diciembre de 1926.

XIV 4 de enero de 1927 a 31 de diciembre de 1927.

XV 3 de enero de 1928 a 28 de diciembre de 1928.

[XVI] 3 de enero de 1929 a 28 de enero de 1930.

Existen 22 legajos con documentación complementaria de los años 1824 a 1930.

(1) El acta de 3 de enero de 1824 se encontraba recogida con la documentación complementaria; se ha incorporado al primer libro. 
1. La información sobre todas las Secciones documentales de los Archivos Estatales, y las materias generales de que tratan, se puede obtener consultando la Base de Datos del Cienso-Guía de Archivos, elaborada por e] CIDA, a través de los PIC (Puntos de Información Cultural) del Ministerio de Cultura, una de cuyas terminales se encuentra situada en la moderna Biblioteca Pública del Estado de Melilla.
CONTEL BAREA, C.: El Archivo de la Presidencia del Gobierno, STUDIA HISTORICA. Salamanca, 1990.

ESCUDERO, J. A.: Los origenes del Consejo de Ministros, Mndrid. Editora Nacional, 1970.

GONZALEZ MARIN̄AS, P.: Génesis y erolución de la Presidencia del Consejo de Ministms en España (1800-1S75), Madrid. 1974.

SAI_AS LARRAZABAL, C.: El Archivo de la Presidencia del Gobierno, Direceión General de Archivos y Bibliotecas, 1973.

SECRETARIA GENERAL TECNICA: Evolución de la Presidencia del Gobierno. 1936-1969, Isegajo 736.

SECRETARIA GENERAL TECNICA: La Presidencia del Cobierno hasta 1963, Legajo 773. 


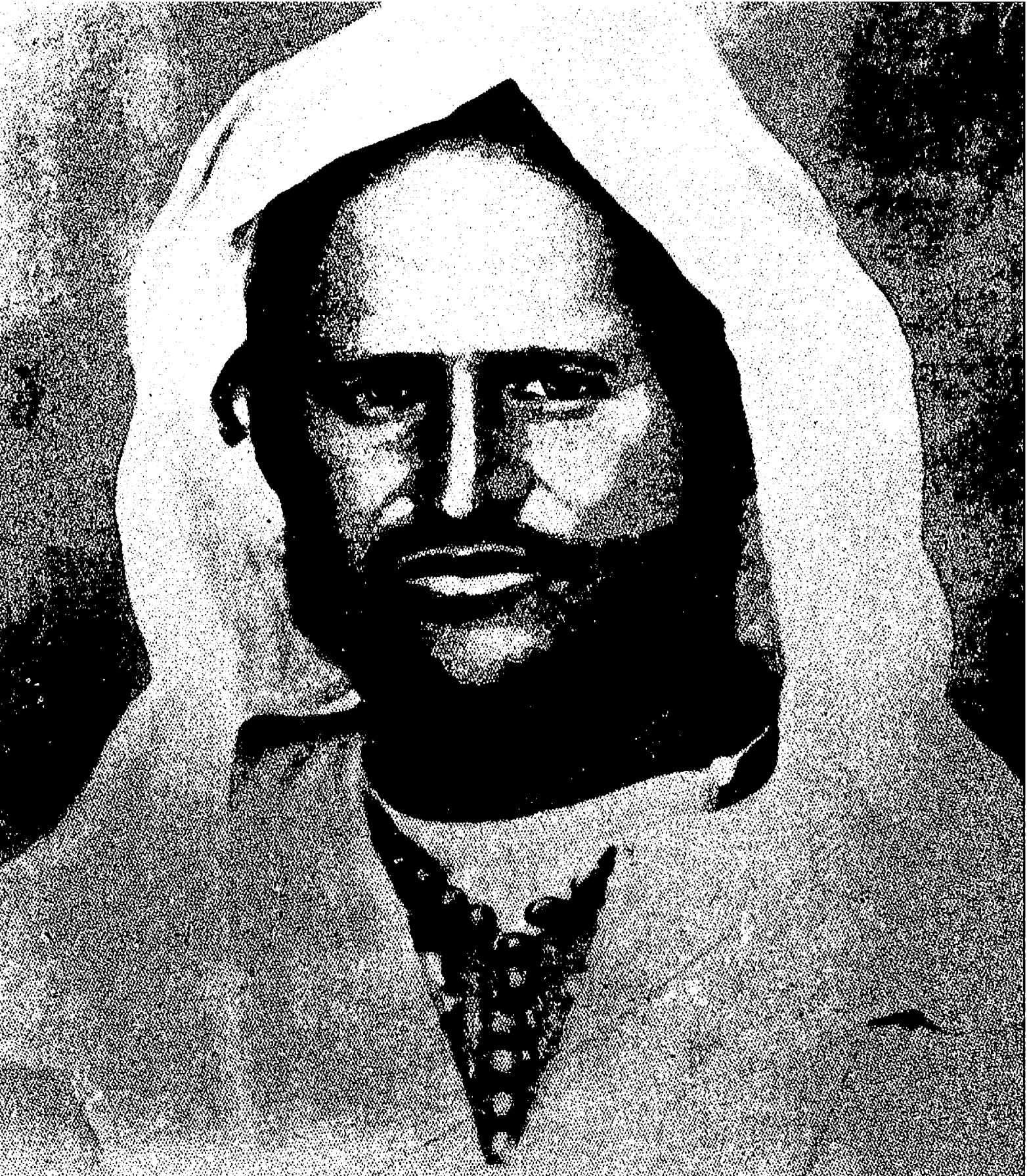

MLFATENFAMADSUJGE H-AEA ENE MATCA A F: NEICON 